\title{
Intramolecular Vibrational Relaxation in Electronically Excited States
}

\author{
R. VOLTZ, A. BOEGLIN and A. A. VILLAEYS \\ Université Louis Pasteur et Centre de Recherches Nucleaires, 67037 Strasbourg \\ CEDEX, France
}

\section{S. H. LIN}

Department of Chemistry, Arizona State University, Tempe, Arizona 85287, USA

A density matrix approach for describing intramolecular dynamics, with special application to vibrational relaxation in excited electronic states, is presented. We derive the master equations governing intramolecular transfer of excitation energy between the states in a zeroth-order basis defined by considering the excitation and detection conditions in the time-resolved experiments. It is shown that, in this formalism, the memory function plays a central role. We note that the form of intramolecular memory is determined by the importance of quantum mechanical mixing of the zeroth-order states. A distinction is made between the subsystems of states with strong and weak mixing properties; while the former account for quasiperiodic character of coherent motion, the latter display a Markovian behavior. The physical conditions fixing the relative importance of quasiperiodic and statistical dynamics in individual systems are discussed. In the application to time resolved intramolecular vibrational relaxation, special consideration is given to the nature of the initially excited doorway states and the intermode couplings. The symmetry restrictions and the possible role of rotational motion in vibrational relaxation are also discussed, before considering the recent results obtained by Zewail et al. with anthracene in the first excited singlet state.

\section{INTRODUCTION}

There has recently been an increased activity in the experimental and theoretical studies of intramolecular vibrational relaxation (IVR) in the presence of other competing processes like fluorescence, isomerization and fragmentation. ${ }^{1-9}$ The time resolved observation of IVR is 
of particular interest, and experimentally most convenient, for vibrational levels in electronically excited states, where the initial level can be prepared by impulsive laser excitation and where temporal evolution can be followed by fluorescence. ${ }^{1,3,4,8,9}$ An interesting example of such experiments, concerned with IVR in the lowest excited singlet state of isolated anthracene molecules, has been reported by Zewail et al. ${ }^{10}$

It is the purpose of this paper to present a density matrix approach for describing intramolecular dynamics, with special application to vibrational relaxation in excited electronic states. In Section 2, we derive the generalized master equations governing intramolecular transfer of excitation energy between the states of the zeroth-order basis (doorway and hallway states), that are determined by the impulsive excitation conditions. It is shown that, in this formalism, the memory function plays the central role in representing the details of intramolecular dynamics. This is analysed in Section 3, by noting that nature and extent of intramolecular memory are determined by the strengths of quantum mechanical coupling and mixing of the zerothorder states. ${ }^{4} \mathrm{~A}$ distinction is therefore made between the subsystems of states that are strongly or weakly mixed: for the former, the quasiperiodic character of coherent motion is reflected by the corresponding terms of the memory function; for the latter, an ultra-short memory, Markoff approximation is generally valid, which leads to a stochastic limit of intramolecular motion. The physical conditions fixing the relative importance of these extreme dynamical modes in individual systems are discussed in a way related to the current understanding of molecular radiationless transitions. We also consider briefly the relationship with other recent classical or quantal descriptions of quasiperiodic and stochastic behavior in molecules. ${ }^{6,7}$ In Section 4, the theoretical treatment is applied to time-resolved IVR in excited electronic states of polyatomic molecules. Here, special consideration must be given to the nature of the initially excited doorway states and the concomitant couplings with the other vibrational modes. The discussion of the mechanisms involving Fermi resonance and Coriolis interaction stresses the importance of symmetry restrictions and the possible role of rotational motion in IVR. As an illustrative example, we consider in some detail the recent results obtained by Zewail et al. with anthracene molecules in the first excited singlet state. ${ }^{10}$ 


\section{MASTER EQUATIONS OF INTRAMOLECULAR DYNAMICS}

The time resolved properties of a molecular system are best described in a zeroth-order representation essentially determined by the experimental excitation and detection conditions. ${ }^{4}$ One thus introduces a model where a clear distinction is made between the doorway states, which can be initially prepared by impulsive excitation and the remaining states (hallway states). These states are not eigenstates of the true molecular Hamiltonian $H$ and are mixed by the residual interaction $H^{\prime}=H-H^{0}$. The resulting excitation energy exchange between the doorway and hallway manifolds, together with relaxation under the influence of the radiation field and eventual heat baths, determines the intramolecular dynamical properties.

In the most simple model, we may consider a single doorway state $|s\rangle$ coupled to a manifold $\{|l\rangle\}$ of hallway states. Only $|s\rangle-|l\rangle$ interaction is taken into account, which means that the hallway-manifold is prediagonalized. ${ }^{11}$ The evolution of the system is then governed by the following equations of motion of the density matrix: ${ }^{12}$

$$
\begin{aligned}
& d \rho_{s s} / d t=-\gamma_{s} \rho_{s s}-i \sum_{l}\left(u_{s l} \rho_{l s}-\rho_{s l} u_{l s}\right)+\delta(t) \\
& d \rho_{l l} / d t=-\gamma_{l} \rho_{l l}-i\left(u_{l s} \rho_{s l}-\rho_{l s} u_{s l}\right) \\
& d \rho_{l s} / d t=-\left(i \omega_{l s}+\gamma_{l s}\right) \rho_{l s}-i u_{l s}\left(\rho_{s s}-\rho_{l l}\right)
\end{aligned}
$$

Here $\gamma_{s}$ and $\gamma_{l}$ represent the total decay rates of the populations of $|s\rangle$ and $|l\rangle$-levels due to the influences of the photon and other baths; $\gamma_{l s}$ denotes the rate constant for relaxation of $(s-l)$ coherence, so that:

$$
\gamma_{l s}=\left[\left(\gamma_{l}+\gamma_{s}\right) / 2\right]+\gamma_{s l}^{*}
$$

where $\gamma_{l s}^{*}$ accounts for pure-dephasing. The other constants are defined as $\omega_{l s}=\left(\varepsilon_{l}-\varepsilon_{s}\right) / \hbar$, where $\varepsilon_{l}$ and $\varepsilon_{s}$ are the energies of the zeroth-order levels, and $u_{l s}=\left\langle l\left|H^{\prime}\right| s\right\rangle / \hbar$. In Eq. (2.1), the last term accounts for initial pulse excitation of the doorway state. It will be useful to consider the Laplace transform of the density matrix

$$
\hat{\rho}(p)=\int_{0}^{\infty} d t \exp (-p t) \rho(t)
$$


so that one has, from Eqs (2.1)-(2.3):

$$
\begin{aligned}
& p \hat{\rho}_{s s}=-\gamma_{s} \hat{\rho}_{s s}-i \sum_{l}\left(u_{s l} \hat{\rho}_{l s}-\hat{\rho}_{s l} u_{l s}\right)+1 \\
& p \hat{\rho}_{l l}=-\gamma_{l} \hat{\rho}_{l l}-i\left(u_{l s} \hat{\rho}_{s l}-\hat{\rho}_{l s} u_{s l}\right) \\
& p \hat{\rho}_{l s}=-\left(i \omega_{l s}+\gamma_{l s}\right) \hat{\rho}_{l s}-i u_{l s}\left(\hat{\rho}_{s s}-\hat{\rho}_{l l}\right)
\end{aligned}
$$

The master equations (ME) describing population transfer between the doorway and hallway manifolds are obtained by eliminating the nondiagonal density matrix elements in Eqs. (2.6) and (2.7):

$$
\begin{aligned}
p \hat{\rho}_{s s}-1 & =-\gamma_{s} \hat{\rho}_{s s}-\sum_{l} \hat{W}_{s l}(p)\left(\hat{\rho}_{s s}-\hat{\rho}_{l l}\right) \\
p \hat{\rho}_{l l} & =-\gamma_{l} \hat{\rho}_{l l}-\hat{W}_{s l}(p)\left(\hat{\rho}_{l l}-\hat{\rho}_{s s}\right) \\
\hat{W}_{s l}(\rho) & =2\left|u_{s l}\right|^{2}\left(p+\gamma_{s l}\right) /\left[\left(p+\gamma_{s l}\right)^{2}+\left(\omega_{s l}\right)^{2}\right]
\end{aligned}
$$

Carrying out the inverse transformation of Eq. (2.9), we get

$$
d \rho_{s s} / d t-\delta(t)=-\gamma_{s} \rho_{s s}-\sum_{l} \int_{0}^{t} d \tau W_{s l}(\tau)\left[\rho_{s s}(t-\tau)-\rho_{l l}(t-\tau)\right]
$$

where $W_{s l}(t)$ is the memory function for the intramolecular radiationless population exchange between the doorway and hallway states; it is the transform of Eq. (2.11):

$$
W_{s l}(t)=2\left|u_{s l}\right|^{2} \exp \left(-\gamma_{s l} t\right) \cos \left(\omega_{s i} t\right)
$$

Eliminating $\hat{\rho}_{l l}$ in Eqs (2.9) and (2.10), we find

$$
\begin{aligned}
\hat{\rho}_{s s}(p) & =\left[p+\gamma_{s}+\hat{W}_{s s}(p)\right]^{-1} \\
\hat{W}_{s s}(p) & =\sum_{l} \frac{\left(p+\gamma_{l}\right) \hat{W}_{s l}(p)}{\left(p+\gamma_{l}\right)+\hat{W}_{s l}(p)}
\end{aligned}
$$

so that we can write:

$$
d \rho_{s s} / d t-\delta(t)=-\gamma_{s} \rho_{s s}-\int_{o}^{t} d \tau W_{s s}(\tau) \rho_{s s}(t-\tau)
$$

Here $W_{s s}(t)$ is the transform of the expression (2.14): it represents the characteristic memory function for intramolecular decay of the 
doorway state due to coupling with the other molecular zeroth-order states.

It is known that under weak interstate mixing and short memory conditions, the generalized ME's can be simplified through a Markoff approximation. ${ }^{13}$ Here the weak mixing condition is expressed as: $:^{4,14}$

$$
\lambda_{l}^{2}=2\left|u_{s l}\right|^{2} /\left[\gamma_{s l}^{2}+\left(\omega_{s l}\right)^{2}\right] \ll 1
$$

and must be considered for each of the convolution terms in the sum over $\{l\}$ of Eq. (2.12). if condition (2.16) holds, the functions $\rho_{s s}(t)$ and $\rho_{l l}(t)$ are slowly variable (i.e., $\left.\rho_{s s}(t-\tau)=\rho_{s s}(t)+0\left(\lambda_{l}^{2}\right), \ldots\right)$ in the convolution integral of interest, which can therefore be expressed as $\left[\int_{0}^{t} d \tau W_{s l}(\tau)\right]\left[\rho_{s s}(t)-\rho_{l l}(t)\right]$; for times larger than the memory decay time $\gamma_{s l}^{-1}$, we may further set $\int_{0}^{t} d \tau W_{s l}(\tau) \sim \int_{0}^{\infty} d \tau W_{s l}(\tau)$. Formally, this amounts to treating the memory function as the distribution

$$
W_{s l}(t)=k_{s l} \delta(t) \quad k_{s l}=\int_{0}^{\infty} d \tau W_{s l}(\tau)=\hat{W}_{s l}(0)
$$

in the ME (2.12), where the corresponding term is now of the ordinary kinetic equation form, with a "rate constant" given by Eqs. (2.17) and (2.11):

$$
k_{s l}=2\left|u_{s l}\right|^{2} \gamma_{s l} /\left[\left(\gamma_{s l}\right)^{2}+\left(\omega_{s l}\right)^{2}\right]
$$

It is interesting to note that the simple rate equation approach is valid whenever $\gamma_{s l} \gg\left|u_{s l}\right|$, i.e., when coherence decay due to external or intramolecular statistical perturbations prevents efficient coherent intramolecular mixing of the zeroth-order states. In strong mixing conditions, when (2.16) is not valid, the exact temporal behavior of the memory must generally be considered.

We are thus led to a natural distinction, in the $|l\rangle$ manifold, of two classes of states

$$
\sum_{l}|l\rangle\left\langle l\left|=\sum_{l^{\prime}}\right| l^{\prime}\right\rangle\left\langle l^{\prime}\left|+\sum_{l^{\prime \prime}}\right| l^{\prime \prime}\right\rangle\left\langle l^{\prime \prime}\right|
$$

which are respectively submitted to strong $\left(l^{\prime}\right)$ and weak $\left(l^{\prime \prime}\right)$ mixing with the doorway state. The ME's thus all include terms with an without intramolecular memory. In Eq. (2.13), the doorway state decay function (2.14) becomes:

$$
\hat{W}_{s s}(p)=\hat{W}_{s s}^{\prime}(p)+\hat{W}_{s s}^{\prime \prime}(p)
$$


where

$$
\hat{W}_{s s}^{\prime}(p)=\sum_{l^{\prime}} \frac{\left(p+\gamma_{l^{\prime}}\right) \hat{W}_{s l^{\prime}}(p)}{\left(p+\gamma_{l^{\prime}}\right)+\hat{W}_{s l^{\prime}}(p)}
$$

accounts for the influence of the strongly mixed hallways states, and exhibits the coherent, quasiperiodic features of intramolecular energy exchange. The influence of the weakly mixed hallway-manifold is represented by

$$
\hat{W}_{s s}^{\prime \prime}(p)=\sum_{l^{\prime \prime}} \frac{\left(p+\gamma_{l^{\prime \prime}}\right) k_{s l^{\prime \prime}}}{p+\gamma_{l^{\prime \prime}}+k_{s l}^{\prime \prime}} \approx \sum_{l^{\prime \prime}} k_{s l^{\prime \prime}}
$$

where the simplified form is justified by remembering that, according to Eqs (2.18) and (2.16), one has $k_{s l^{\prime \prime}}=\lambda_{l^{\prime \prime}}^{2} \gamma_{s l^{\prime \prime}} \ll \gamma_{s l^{\prime \prime}}$, i.e., $k_{s l^{\prime \prime}} \ll\left(p+\gamma_{l}\right)$, in general; the constant value of expression (2.22) may be regarded as the rate constant

$$
k_{s}=\sum_{l^{\prime \prime}} k_{s l^{\prime \prime}}
$$

for Markovian intramolecular energy transfer from the doorway to the hallway states. In the following, it will be convenient to define

$$
\Gamma_{s}=\gamma_{s}+k_{s}
$$

as the total doorway decay constant, and to consider the ME's (2.13) and $(2.15)$ in the forms:

$$
\begin{gathered}
\hat{\rho}_{s s}(p)=\left[p+\Gamma_{s}+\hat{W}_{s s}^{\prime}(p)\right]^{-1} \\
d \rho_{s s} / d t-\delta(t)=-\Gamma_{s} \rho_{s s}-\int_{0}^{t} d \tau W_{s s}^{\prime}(\tau) \rho_{s s}(t-\tau)
\end{gathered}
$$

where $W_{s s}^{\prime}(t)$ is the transform of expression (2.21).

The experimental observables are directly related to the density matrix elements given by these ME's. In photophysical measurements, for example, the fluorescence from the initially excited doorway state (prerelaxation or resonance fluorescence) has the intensity and quantum yield respectively given by:

$$
I(t)=\gamma_{s}^{(r)} \rho_{s s}(t) \quad Q=\gamma_{s}^{(r)} \int_{0}^{\infty} d t \rho_{s s}(t)=\gamma_{s}^{(r)} \hat{\rho}_{s s}(0)
$$

where $\gamma_{s}^{(r)}$ denotes the fluorescence rate constant from the $|s\rangle$-level. 
Similar quantities may be defined for fluorescence from the hallwaystates ("relaxed" fluorescence):

$$
I_{l}^{\prime}(t)=\gamma_{l}^{(r)} \rho_{l l}(t) \quad Q_{l}^{\prime}=\gamma_{l}^{(r)} \hat{\rho}_{l l}(0)
$$

which requires consideration of Eq. (2.10) and its Laplace transform; it is, in particular, verified that the quantum yield of the relaxed fluorescence components is given by

$$
\boldsymbol{Q}_{l}^{\prime}=\gamma_{l}^{(r)}\left[k_{s l} /\left(\gamma_{l}+k_{s l}\right)\right] \hat{\rho}_{s s}(0)
$$

where $k_{s l}$ is the intramolecular energy exchange rate (2.18).

\section{MEMORY FUNCTIONS AND DYNAMICAL MODES}

The dynamical modes amenable to experimental observations are determined by the memory functions of the foregoing ME's. The various limiting cases of intramolecular-either electronic or vibrational ${ }^{14,4,9}$ - relaxation can hence be discussed in terms of limiting forms of these functions, with special consideration for the doorway state decay function $W_{s s}$ as given by Eqs. (2.20)-(2.23).

\section{The small-molecule limit}

It is characterized by level densities so small as to satisfy the "weak coupling" condition ${ }^{4,14}$

$$
\left|u_{s l}\right| \ll \delta \varepsilon_{l}
$$

where $\delta \varepsilon_{l}$ represents the spacing of hallway levels. The usual term denoting this limiting case is obviously too restrictive since the condition (3.1) is not necessarily related to the molecular size and may be equally valid for large molecules with sufficiently small excitation energies.

In the absence of accidental degeneracy, all the hallway states are weakly mixed with the doorway state, so that $\hat{W}_{s s}(p)=\hat{W}_{s s}^{\prime \prime}(p)=k_{s}$, following Eqs. (2.22)-(2.23). The intramolecular decay constant $k_{s}$ is usually very small compared to $\gamma_{s}$. This is a relatively trivial situation where the initially excited state has a "local" behavior only slightly influenced by intramolecular coupling: it decays exponentially with the decay constant (2.24) and is responsible for spectra of discrete nonoverlapping levels. 
In the presence of accidental quasidegeneracy, the $|l\rangle$-level nearest to the $|s\rangle$-level is so that $\omega_{s l} \leqslant\left|u_{s l}\right|$. This level then contributes alone to the expression (2.21) of the quasi-periodic memory function $\hat{W}_{s s}^{\prime}(p)$; we thus obtain:

$$
\hat{\rho}_{s s}(p)=\left[p+\Gamma_{s}+\frac{\left(p+\gamma_{l}\right) \hat{W}_{s l}(p)}{p+\gamma_{l}+\hat{W}_{s l}(p)}\right]^{-1}
$$

for Eq. (2.25). In situations where no efficient relaxation takes place $\left(\left|u_{s l}\right| \gg \gamma_{s l}\right)$, Eq. (3.2) predicts coherent behavior with quantum beats: assuming, for simplicity, that no pure dephasing is occurring i.e., $\gamma_{s l}^{*}=0$ in Eq. (2.4) and that $\Gamma_{s}=\gamma_{l}=\gamma$, one gets:

$$
\begin{aligned}
\hat{\rho}_{s s}(p) & =\frac{\omega_{s l}^{2}+2\left|u_{s l}\right|^{2}}{\Lambda_{s l}^{2}} \frac{1}{p+\gamma}+\frac{2\left|u_{s l}\right|^{2}}{\Lambda_{s l}^{2}} \frac{p+\gamma}{(p+\gamma)^{2}+\Lambda_{s l}^{2}} \\
\Lambda_{s l}^{2} & =\omega_{s l}^{2}+4\left|u_{s l}\right|^{2}
\end{aligned}
$$

The beats may be observed through time-resolved intensity measurements of resonance fluorescence (cf. Eqs. 2.27), given by

$$
I(t)=\gamma_{s}^{(r)} \exp (-\gamma t)\left[\frac{\omega_{s l}^{2}+2\left|u_{s l}\right|^{2}}{\Lambda_{s l}^{2}}+\frac{2\left|u_{s l}\right|^{2}}{\Lambda_{s l}^{2}} \cos \left(\Lambda_{s l} t\right)\right]
$$

These results have been applied to analyze quantum beats observed with molecular systems in the gas and condensed phases; ${ }^{15,16}$ they will be used below to discuss the data recently obtained for anthracene with supersonic jet techniques. ${ }^{10}$ For the fluorescence quantum yield (2.27), we obtain:

$$
Q=\gamma_{s}^{(r)}\left[\Gamma_{s}+k_{s l} \gamma_{l} /\left(\gamma_{l}+k_{s l}\right)\right]^{-1}
$$

If strong mixing conditions apply for the two interacting levels, it is seen that $k_{s l} \gg \gamma_{l}$, so that $Q=\gamma_{s}^{(r)} /\left(\Gamma_{s}+\gamma_{l}\right)$; one hence recovers the intuitively obvious result that light emission is determined equally by the two strongly mixed zeroth-order states.

\section{The large-molecule limit}

Here the density of states is so large that the "strong coupling" condition $^{4,14}$

$$
\left|u_{s l}\right| \gg \delta \varepsilon_{l}
$$

is generally valid. 
In situations of efficient coherence and population decay, where $\gamma_{s l}>\left|u_{s l}\right|$, the hallway states may however satisfy the weak mixing conditions (2.16), even if (3.7) holds. According to the Eqs. (2.22) and (2.23), the decay memory function is then constant and given by:

$$
k_{s}=\sum_{l} 2\left|u_{s l}\right|^{2} \gamma_{s l} /\left[\left(\gamma_{s l}\right)^{2}+\left(\omega_{s l}\right)^{2}\right]
$$

As usual in the treatments of the "statistical limit" in radiationless transitions, ${ }^{4,14}$ this sum of overlapping Lorentzian functions can be evaluated as an integral over a hallway quasicontinuum characterized by a mean level density $\left\langle\left(\delta \varepsilon_{l}\right)^{-1}\right\rangle$ and coupling constant $\left\langle\left|u_{s l}\right|^{2}\right\rangle$; the result is:

$$
k_{s}=2 \pi\left\langle\left|u_{s l}\right|^{2}\right\rangle\left\langle\left(\delta \varepsilon_{l}\right)^{-1}\right\rangle
$$

Resonance fluorescence intensity and quantum yield are of the simple forms

$$
I(t)=\gamma_{s}^{(r)} \exp \left(-\Gamma_{s} t\right) \quad Q=\gamma_{s}^{(r)} / \Gamma_{s}
$$

which, together with broad spectral lines, are usually found in most of the polyatomic molecules at high excitation energies, not isolated from medium effects. We may note that this case corresponds to an extreme stochastic situation, where the doorway state is connected via Markovian interaction with a large number of overlapping hallway-levels.

In situations with relaxation so weak that $\gamma_{s l}<\left|u_{s l}\right|$, a relatively large number of hallway states can be strongly mixed with the doorway level; this corresponds to the "intermediate cases" of radiationless transitions in molecules, ${ }^{4,9,14}$, which must be treated beyond the Markoff approximation, by including also the quasiperiodic features through a memory function (2.21). The doorway population $\rho_{s s}(t)$ or the resonance fluorescence intensity then evolve differently at short and long times.

According to the properties of Laplace transforms, the short time behavior of $\rho_{s s}(t)$ is determined by $\hat{\rho}_{s s}(p)$ at large values of $p$. For $p \gg\left|u_{s l}\right|$ (i.e., $t \ll\left|u_{s l}\right|^{-1}$ ), we readily verify that the memory function $\hat{W}_{s s}^{\prime}(p)$ defined by Eq. $(2.21)$ can safely be approximated as:

$$
\hat{W}_{s s}^{\prime}(p) \approx \sum_{l^{\prime}} \hat{W}_{s l^{\prime}}(p)=\sum_{l^{\prime}} 2\left|u_{s l^{\prime}}\right|^{2} \frac{p+\gamma_{s l^{\prime}}}{\left(p+\gamma_{s l^{\prime}}\right)^{2}+\left(\omega_{s l^{\prime}}\right)^{2}}
$$


Due to the large values of $p\left(p \gg \delta \varepsilon_{l}\right)$, this can be evaluated as an integral, in the same way as for expression (3.8); the result is the same as Eq. (3.9), which shows that the initial decay of $\rho_{s s}(t)$ and $I(t)$ in the intermediate case is identical to the exponential decay (3.10) in the statistical limit. At large times, the evolution of $\rho_{s s}(t)$ depends on the behavior of $\hat{\rho}_{s s}(p)$ as $p \rightarrow 0$. It is seen that, in this limit, one has $\hat{W}_{s l^{\prime}}(p) \gg p+\gamma_{l^{\prime}}$ for the strongly mixed $\left|l^{\prime}\right\rangle$-levels, satisfying the relationship

$$
2\left|u_{s l^{\prime}}\right|^{2} /\left(\gamma_{l^{\prime} s}^{2}+\omega_{l^{\prime} s}^{2}\right) \gg\left(\gamma_{l^{\prime}} / \gamma_{l^{\prime} s}\right)
$$

Denoting by $N$ the number of such levels, we may then set

$$
\hat{W}_{s s}^{\prime}(p)=\sum_{l^{\prime}}\left(p+\gamma_{l^{\prime}}\right)=N\left(p+\gamma_{l}\right)
$$

so that Eq. (2.25) becomes:

$$
\hat{\rho}_{s s}(p)=(N+1)^{-1}\left[p+\left(\Gamma_{s} / N+1\right)+\left(N \gamma_{l} / N+1\right)\right]^{-1}
$$

This indicates exponential decay at long times, but with a time constant given as the weighted average of the individual zeroth-order contributions in the strongly mixed energy band:

$$
\Gamma=\Gamma_{s} /(N+1)+N \gamma_{l} /(N+1)
$$

The biexponential decay law, with different decay constants at short and long times, is typical for the intermediate cases: ${ }^{4,9,14}$

$$
\begin{array}{ll}
I(t)=\gamma_{s}^{(r)} \exp \left(-\Gamma_{s} t\right) & t<\left|u_{s l}\right|^{-1} \\
I(t)=\gamma_{s}^{(r)} \exp (-\Gamma t) /(N+1) & t>\left|u_{s l}\right|^{-1}
\end{array}
$$

where $\Gamma_{s}$ and $\Gamma$ are respectively defined by Eqs. (2.24) and (3.15). The resonance fluorescence quantum yield is obtained from Eqs. (2.27) and (2.25), by noting that $\hat{W}_{s s}^{\prime}(0)=N \gamma_{l}$, according to Eq. (3.13):

$$
Q=\gamma_{s}^{(r)} /\left(\Gamma_{s}+N \gamma_{l}\right)
$$

This means that, in a way similar to the two strongly interacting levels in quantum beats (cf. supra), the fluorescence efficiency is determined by the decay constants of all the strongly mixed zeroth-order states.

The essential difference between the statistical limit and the intermediate cases must be stressed. While at short times, the observed 
doorway state decay is similar, it must however be attributed to fundamentally different processes: strong irreversible relaxation, with $\gamma_{s l} \gg \delta \varepsilon_{l}$, in the statistical systems, quantal intramolecular wavepacket dephasing, characterized by uncertainty broadening $\left(p \approx t^{-1}\right) \gg \delta \varepsilon_{l}$ at short times in the intermediate cases. The difference is best exhibited in long time experiments, either through observation of the long time decay (3.17) in the intermediate cases only, or through the different forms (3.10) and (3.18) of the fluorescence quantum yields.

It may be of interest to relate the present quantum mechanical results to the classical approaches of interacting resonances in multidimensional system. ${ }^{6,7,17,18}$ The boundary, $\left(u_{s l} / \delta \varepsilon_{l}\right)=1$, which separates the small and large molecule cases has an analogue in the "stochasticity boundary" defined by Chirikov in the classical description. ${ }^{18}$ Below that boundary, motion is generally regular and periodic as for the quantum mechanical small molecule limit. Above the boundary, when a condition comparable to (3.7) is valid, the classical motion is generally found to be stochastic. According to our results, this is not necessarily the case for the corresponding quantum mechanical large molecule limit. A meaningful comparison of classical and quantal stochasticity is possible by associating stochastic motion to Markovian behavior. Similarity of classical and quantum mechanical dynamics with respect to stochasticity is then expected when the frequency (or $p$ ) dependent memory functions $\hat{W}_{s s}(p)$ are smooth enough, i.e., in the statistical limit and the intermediate cases at small times; but definite deviations are expected with the intermediate cases at long times, where $\hat{W}_{s s}(p)$ is no longer constant (cf. Eq. (3.13)). Such long time deviations of the quantum mechanical results from the classical results were indeed noted in numerical calculations on a simple model ${ }^{19}$ and further discussed by Rice and Kosloff $^{20}$ and by Zaslavskii. ${ }^{21}$

\section{INTRAMOLECULAR VIBRATIONAL RELAXATION}

The analysis presented in Sections 2 and 3 is general, and may be applied to any intramolecular relaxation process, either electronic or vibrational. We focus here on the temporal properties of IVR in an electronically excited state, bearing in mind anthracene as an illutrative example. 


\section{The basis of representation}

To begin, it is necessary to specify the basis of representation in terms of which intramolecular vibrational dynamics are defined and described.

The doorway states are those initially prepared in the electronic radiative transition (e.g., $S_{0} \rightarrow S_{1}$ ) exciting the molecule. In the case of symmetry allowed transitions, ${ }^{24}$ such vibrational modes result from the change in the equilbrium configuration of the molecule, and are generally totally symmetric $\left(a_{1 g}\right)$. These Franck-Condon states are responsible for the quasilinear absorption and fluorescence spectra observed with polyatomic molecules in vapor or in low temperature matrices. ${ }^{3,5,22,23}$ Fluorescence from these initially excited doorway states is referred to as direct or resonance fluorescence; it enables one to measure the quantities (2.27), which represent the rate and extent of doorway state decay.

The manifold of hallway states includes all the states not excited by the electronic symmetry allowed transition. ${ }^{24}$ They correspond to the non-totally symmetric species (fundamentals and overtones) and may include the majority of low frequency oscillators, hence forming a relatively dense manifold. The radiative vibronic transitions $\left(S_{1} \rightarrow S_{0}\right)$ with initial non-totally symmetric vibrational modes take generally place without shift of the equilibrium position, so that the vibrational occupation number does not change. But there is usually a notable change of the vibration frequency, which is generally larger in the electronic ground state $S_{0}{ }^{25}$ As a result, the corresponding spectral lines are red-shifted with respect to the resonance fluorescence lines from the isoenergetic doorway levels, that usually involve only minor frequency changes. The diffuse, red-shifted fluorescence spectra in polyatomic molecular systems are thus usually attributed to radiative transitions from the hallway manifold: ${ }^{3-5}$ this relaxed fluorescence component allows one to monitor the population of the hallway states and hence to follow IVR; the corresponding observables are given by Eqs. (2.28) and (2.29).

For interstate coupling of the vibrational doorway and hallway states, the most important mechanisms are usually taken to be Fermi resonance and Coriolis interaction. ${ }^{26}$ If the two interacting modes have frequencies so different that they can be separated in an adiabatic approximation, it is convenient to represent intermode coupling by 
non-adiabaticity operators, similar to those describing Born-Oppenheimer coupling of vibronic states. ${ }^{27}$

Fermi resonance is caused by anharmonicity and occurs between close-lying doorway $(s)$ and hallway $(l)$ levels, which must have the same vibrational symmetries. The first anharmonic term which can couple the totally symmetric $|s\rangle$-level (mode coordinate $q_{s}$ ) to a non-totally symmetric $|l\rangle$-level (coordinate $Q_{l}$ ) has then the form:

$$
H_{a n h}^{\prime}=\lambda_{s l} q_{s} Q_{l}^{2}
$$

This interaction can create even overtones in the hallway-modes at the expense of the doorway quanta. Experimental evidence for the dynamical role of such couplings was noted for IVR in triatomic molecules; ${ }^{28}$ further theoretical aspects have been investigated by Heller et al. ${ }^{29}$

Coriolis interaction is caused by terms in the rotation-vibration kinetic energy operator of the molecular Hamiltonian, depending linearly on both a vibrational $(\mathbf{m})$ and a rotational $(\mathbf{M})$ angular momentum operator: ${ }^{26,27,30}$

$$
H_{\mathrm{Cor}}=-\sum_{\mu}\left(m_{\mu} M_{\mu}\right) / I_{\mu}
$$

Here $m_{\mu}$ and $M_{\mu}$ represent respectively the molecule fixed components $(\mu=x, y, z)$ of the vibrational and angular momenta; $I_{\mu}$ is the moment of inertia. The vibrational angular momentum is generated by cooperative action of the two modes $|s\rangle$ and $|l\rangle$, which may be expressed by

$$
m_{\mu}=\zeta_{s l}^{(\mu)}\left(q_{s} P_{l}-p_{s} Q_{l}\right)
$$

in terms of the conjugate coordinates of the interacting oscillators. Unlike the purely vibrational interaction (4.1), Coriolis interaction hence involves the rotational motions. While the interacting states must have the same rovibrational symmetry, coupling is however possible between states of different vibrational symmetries: the product of their species must contain the species of a rotation; this means that, in the presence of the totally symmetric doorway modes, the coupled hallway modes have the same species as the rotation. For example, in molecules belonging to the $D_{2 h}$ symmetry group, only the $b_{1 \mathrm{~g}}, b_{2 \mathrm{~g}}$ and $b_{3 \mathrm{~g}}$ vibrations are Coriolis active hallway states. From Eq. (4.2), it is readily verified that the interaction conserves the total 
rotational angular momentum quantum number $J$; also the coupling matrix elements depend on the rotational quantum numbers, increasing generally with rotational energy. ${ }^{30}$

To illustrate the foregoing arguments, we consider the case of IVR for the vibrational levels in the first excited singlet state of anthracene (symmetry $D_{2 h}, 66$ normal modes). Here, the electronic state is prepared by an allowed radiative process, so that the vibrational doorway states are totally symmetric; these modes involve in-plane motions, ${ }^{31,32}$ and the low-energy part of the spectrum is mainly determined by $\mathrm{C}-\mathrm{C}-\mathrm{C}$ bending modes, with fundamental frequencies at 390 and $625 \mathrm{~cm}^{-1}$, and by the $1400 \mathrm{~cm}^{-1} \mathrm{C}-\mathrm{C}$ stretching vibration. ${ }^{22,23}$ The hallway manifold includes in principle the 54 non-totally symmetric vibrations; at relatively low vibrational energies, a special role may however be expected from the lowest frequency modes $\left(\omega<1000 \mathrm{~cm}^{-1}\right)$, for example, the $b_{2 g}$ and $b_{3 g}$ out-ofplane bending vibrations, ${ }^{31,33}$ Such modes are known to have frequency changes on electronic transitions as much as $30 \%{ }^{34}$ so that non-negligible red shift of the relaxed fluorescence spectrum should occur. Regarding the coupling mechanisms between the doorway and hallway levels, anharmonicity certainly involves a complex set of weakly mixed hallway configurations including various overtones and combinations of the above mentioned low frequency vibrations; these configurations pertain to the $\left\{\left|l^{\prime \prime}\right\rangle\right\}$ manifold in Eq. (2.19). Strong mixing via Fermi resonance could eventually take place between the fundamental of the $1400 \mathrm{~cm}^{-1} a_{1 \mathrm{~g}}$ stretching mode $(|s\rangle$-level $)$ and the $n=2$ state of the $698 \mathrm{~cm}^{-1}$ out-of-plane bending mode $\left(\left|l^{\prime}\right\rangle\right.$-level in Eq. (2.19) observed in the Raman spectra ${ }^{33}$. For Coriolis coupling, the hallway states of possible importance are $b_{1 \mathrm{~g}}, b_{2 \mathrm{~g}}$ and $b_{3 \mathrm{~g}}$ vibrations. As for anharmonic interaction, the $b_{2 g}$ and $b_{3 g}$ out-of-plane bending modes undergo generally weak mixing with the higher energy $a_{1 \mathrm{~g}}$ doorway modes; stronger mixing may eventually be envisaged for the $b_{1 \mathrm{~g}} \mathrm{C}-\mathrm{H}$ in -plane bending mode, which has energies comparable to the $1400 \mathrm{~cm}^{-1}$ doorway state. ${ }^{33}$

\section{Time resolved observation}

The properties of IVR in electronic excited states of polyatomic molecules, observed by time-resolved fluorescence spectroscopy, can be analysed by means of the foregoing theoretical model, only if the 
basic assumption of an impulsive excitation of an isolated doorway state is justified. According to recent spectroscopic studies of isolated large molecules, this is usually the case up to vibrational excess energies of $2000 \mathrm{~cm}^{-1}$, where a congested vibrational quasicontinuum sets on. ${ }^{35}$ Beyond such energies, coherent excitation of several doorway levels is difficult to avoid, a situation which must be treated by an extended theoretical description. ${ }^{36}$ Among the experimental results of interest in the present context, those published by Zewail et al. on anthracene are particularly meaningful; they indeed combined coherent broad-band excitation, with subnanosecond resolution, and supersonic jet techniques, so that well-defined vibrational doorway states could be impulsively prepared, in the absence of the cumbersome effects of sequence congestion. ${ }^{10}$

Upon $S_{0} \rightarrow S_{1}$ laser excitation, with increasing vibrational excess energies, the dynamical behaviors of the small and large molecule limits, described in Section 3, have been successively observed. For vibrational energies less than $1400 \mathrm{~cm}^{-1}$, the detected fluorescence has the characteristic features of resonance radiation with sharp lines corresponding to the familiar optically active $\left(1400\right.$ and $390 \mathrm{~cm}^{-1}$ ) $a_{1 \mathrm{~g}}$ vibrations of anthracene; this corresponds to a typical small molecule behavior, where the doorway states do not interact with the hallway manifold. For excitation energies at $3439 \AA$ and shorter wavelengths, i.e., with excitation energies larger than $1400 \mathrm{~cm}^{-1}$, when the totally symmetric $\mathrm{C}-\mathrm{C}$ stretching mode is excited, it is found that the relative intensity of the narrow hot bands is strongly reduced, while the red-shifted diffuse spectral components appear with a relative importance increasing with the excess energy. This indicates that at such energies, the large molecule limit is reached where vibrational intermode relaxation progressively sets in according to the statistical limit, for which eqs. (3.8)-(3.10) apply; the weakly mixed hallway modes involved are presumably the low energy out-ofplane motions mentioned above.

In the neighborhood of the $1400 \mathrm{~cm}^{-1}$ doorway excitation energy, where Markovian redistribution begins to be detectable, Zewail et al. also observed a quantum beat pattern, indicating strong mixing of two levels; this corresponds to an interesting small molecule case where, in addition to the Markovian contributions, a quasiperiodic memory function (3.11) with a single dominating term must be considered. In practice, the beats are best detected in the extreme blue 
part of the emission spectrum, where resonance fluorescence from the doorway state is not overlapped by the relaxed components. The intensity can be described by Eq. (3.5), with numerical values that are best determined by a Fourier analysis of the published curves; ${ }^{15,16}$ we thus found $\gamma^{-1}=8.2 \mathrm{~ns}, \Lambda_{s l}=3.1 \times 10^{9} s^{-1}, 2\left|u_{s l}\right|^{2} /\left[\omega_{s l}^{2}+2\left|u_{s l}\right|^{2}\right]=$ 0.1 , so that $\left|u_{s l}\right|=3.5 \times 10^{8} \mathrm{~s}^{-1}$ or equivalently, $\left|u_{s l}\right|=1.2 \times 10^{-2} \mathrm{~cm}^{-1}$. As noted by Rettschnick, ${ }^{3}$ intermode coupling energies are indeed expected to be of this order of magnitude, since larger values would lead to vibrational bandwidths larger, and to electronic spectra in low temperature matrices more complex than usually observed for large molecules. As stated before, a possible candidate for the resonant $|s\rangle-|l\rangle$ interaction is Fermi resonance between the doorway $1400 \mathrm{~cm}^{-1}$ mode and the overtone of the out of plane bending mode with frequency $\omega_{l}=698 \mathrm{~cm}^{-1}$. Coriolis interaction appears as less probable in view of the apparent insensitivity of the results on the rotational states; rotational quantum numbers larger than 1 would indeed lead to large fluctuations of the coupling matrix elements and hence to "coarse graining" of the quasiperiodic memory function (3.11) with quenching of the beats.

\section{References}

1. C. S. Parmenter, J. Phys. Chem. 86, 1735 (1982).

2. R. E. Smalley, J. Phys. Chem. 86, 3504 (1982).

3. R. Rettschnick, in: Radiationless Transitions, ed. S. H. Lin (Academic Prese, New York, 1980) p. 185.

4. A. Tramer and R. Voltz, Excited States 4, 281 (1979).

5. B. S. Neporent, Opt. Spectrosc. 32, 133 (1972).

6. D. W. Noid, M. L. Koszykowski and R. A. Marcus, Ann. Rev. Phys. Chem. 32, 267 (1981).

7. S. A. Rice, Adv. Chem. Phys. 47, 117 (1981).

8. C. Tric, Chem. Phys. 14, 189 (1976).

9. K. F. Freed and A. Nitzan, J. Chem. Phys. 73, 4765 (1980).

10. A. H. Zewail, W. R. Lambert, P. M. Felker, J. Perry and W. Warren, J. Phys. Chem. 86, 1184 (1982).

11. The validity of such a model is considered in Refs. 8 and 9.

12. S. H. Lin and H. Eyring, Proc. Nat. Acad. Sci. U.S. 74, 3623 (1977).

13. R. Zwanzig, in: Lectures in Theoretical Physics, Vol. 3, ed. W. E. Brittin, et al. (Interscience Pub., New York, 1961) p. 106.

14. R. Voltz, in: Organic Molecular Photophysics, Vol. 2, ed. J. B. Birks (John Wiley, London, 1975) p. 217.

15. W. Henke, H., L. Selzle, T. R. Hays, S. H. Lin and E. W. Schlag, Chem. Phys. Lett. 77, 448 (1981).

16. A. A. Villaeys, S. H. Lin, A. Boeglin and J. Klein, Chem. Phys. Lett. 93, 293 (1982). 
17. B. V. Chirikov, Phys. Repts. 52, 263 (1979).

18. G. M. Zaslavskii and B. V. Chirikov, Sov. Phys. Usp. 14, 549 (1972).

19. G. Casati, B. V. Chirikov, F. M. Israilev and J. Ford, in: Stochastic Behaviour in Classical and Quantum Hamiltonian Systems, eds G. Casati and J. Ford (Springer, Berlin, 1979) Lect. Notes Phys. 93, 334 (1979).

20. S. A. Rice and R. Kosloff, J. Phys. Chem. 86, 2153 (1982).

21. G. M. Zaslavskii, Sov. Phys. Usp. 24, 725 (1981).

22. S. O. Mirumyants, E. Vandynkov and Y. S. Demchuk, Opt. Spectrosc. 38, 25 (1978).

23. G. Small, J. Chem. Phys. 52, 656 (1970).

24. An extension of the following arguments to symmetry forbidden (Herzberg-Teller) electronic transitions is straightforward; cf. e.g., Ref. 25.

25. E. F. Sheka, Sov. Phys. Usp. 14, 484 (1972).

26. G. Herzberg, Infrared and Raman Spectra of Polyatomic Molecules (Van NostrandReinhold, Princeton, 1945) pp. 466-468.

27. S. H. Lin, Chem. Phys. Lett. 70, 492 (1980).

28. L. E. Brus and V. E. Bondybey, in: Radiationless Transitions, ed. S. H. Lin (Academic Press, New York, 1980) p. 259.

29. E. J. Heller, E. B. Stechel and M. J. Davis, J. Chem. Phys. 73, 4720 (1980).

30. S. H. Lin, Zhang Xing-Guo, Qian Zhi-Ding, Li Xing-Wen and H. Eyring, Proc. Natl. Acad. Sci. USA 79, 1356 (1982).

31. E. P. Krainov, Opt. Spectrosc. 16, 532 (1964).

32. K. Ohno, J. Mol. Spectrosc. 77, 329 (1979).

33. N. Abasbegovič, N. Vukovič and L. Colombo, J. Chem. Phys. 41, 2575 (1964).

34. R. W. Munn and W. Siebrand, J. Chem. Phys. 52, 47 (1970).

35. A. Amirev, U. Even and J. Jortner, Opt. Commun. 32, 266 (1980).

36. R. Voltz, to be published. 